\title{
Selective Detection of Secondary Electrons in an SEM using a Multi-Channel Detector
}

\author{
Yasuhiro Shirasaki and Momoyo Enyama \\ Hitachi, Ltd., Kokubunji-shi, Tokyo, Japan
}

In a scanning electron microscope (SEM), the contrast of its images depends on the energies and the emission angles of the signal electrons detected by the detectors. This is because the emission energy of a signal electron is dependent on the material and is likely to be emitted in the direction normal to the surface. Therefore, selective detection of signal electrons can lead to the extraction of material and topological information from SEM images. For example, an annular detector placed below the objective lens is often used to detect signal electrons emitted off the optical axis. By selectively detecting secondary electrons (SE) or back-scattered electrons (BSE) by their emission angles, material contrast can be obtained [1]. On the other hand, through-the-lens (TTL) detector placed above the objective lens to selectively detect SE that are emitted along the optical axis have been shown to be important in observing high aspect-ratio semiconductor devices [2]. However, selective detection of SE using TTL detectors is difficult due to the fact that the secondary optics required often interferes with the primary optics. In this report, we overcame this problem by using a beam separator to separate the SE from the primary optics before detecting it with a multi-channel detector to perform selective detection of signal electrons.

A schematic of the SEM we prototyped is shown in Fig. 1. A semi-in-lens objective lens and a retarding voltage were used to collect and accelerate all the SE upwards into a secondary beam. A beam separator placed above the objective lens separated the secondary beam from the primary optics, and a secondary optics projected the secondary beam profile onto a detector. To demonstrate selective detection of signal electrons, commercially available two-dimensional 16-channel detector was used. The secondary optics was designed so the middle two rows of the 16-channel detector can perform emission angle selective detection on SE with energy less than $6 \mathrm{eV}$. This allows about $80 \%$ of SE to be used for the emission angle selective detection. SE with energy greater than $6 \mathrm{eV}$ is detected by all the channels. Accordingly, the top and bottom rows of the 16-channel detector detect only SE with energy greater than $6 \mathrm{eV}$, which can be useful in identifying charging that shifts the emission energy of signal electrons by a few eV. Therefore, the 16-channel detector can be used for selective detection of signal electrons by their energies and emission angles simultaneously.

An example of 16 SEM images obtained simultaneously using the 16-channel detector is shown in Fig. 2. The sample is a particle on a patterned Si substrate. As the images show, different shadowing is observed around the particle between different detector channels. The arrows indicate the shadowing direction we expect for detectors $05 \sim 12$, which we have calculated using electron optical simulation of the secondary beam trajectory. The observed and calculated direction of the shadowing are in good agreement, indicating that the SE are detected by their emission angles as designed. Of the 8 other detectors, detectors 01 04 are expected to detect more of the SE with higher energies and their images show an enhanced contrast between the particle and the substrate.

In conclusion, we have demonstrated selective detection of SE with a 16-channel TTL detector. 

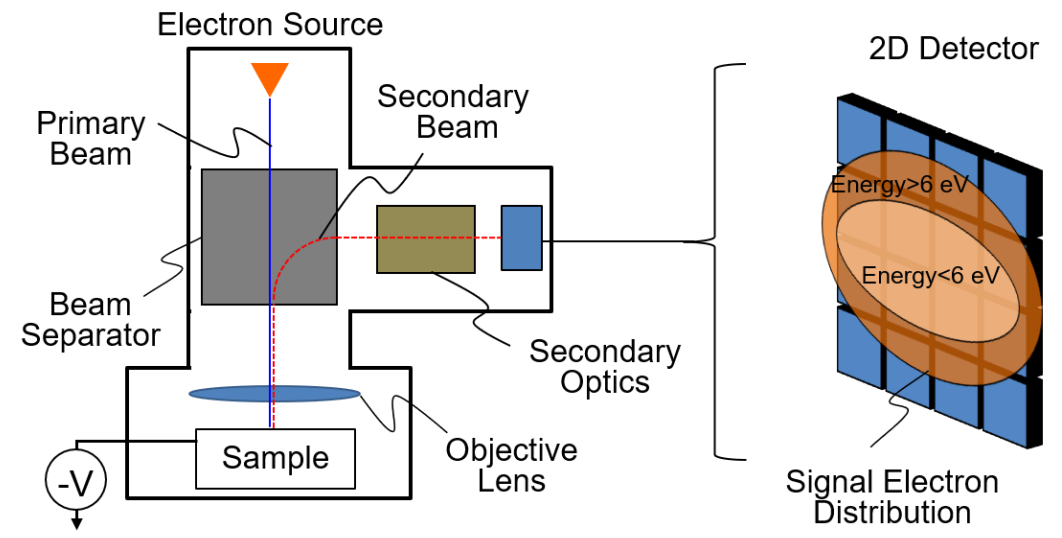

Multi-Channel SEM Images

Figure 1. Schematic of the SEM with a 16-channel detector.

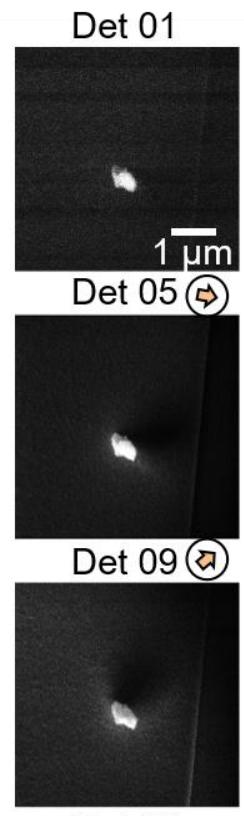

Det 13

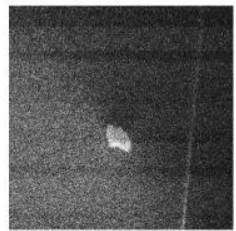

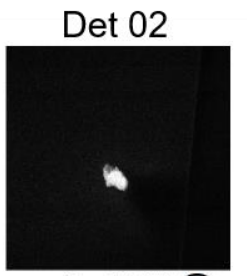

Det 06 (9)

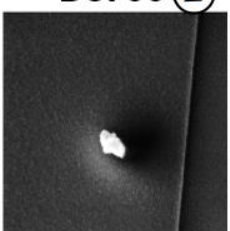

Det 10 (2)

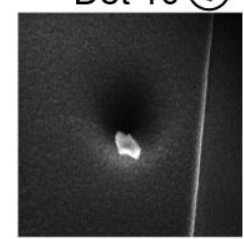

Det 14

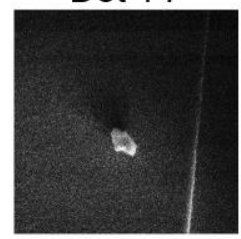

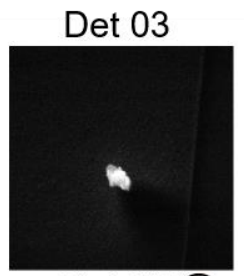

Det 07 (3)

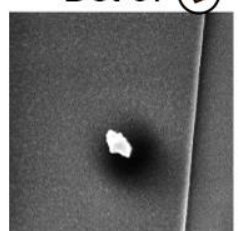

Det 11 (

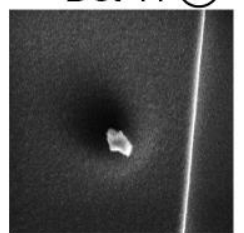

Det 15

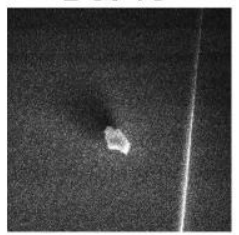

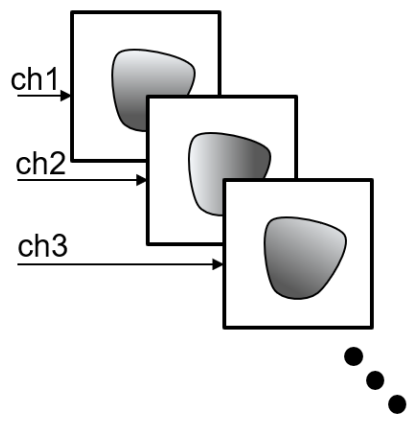

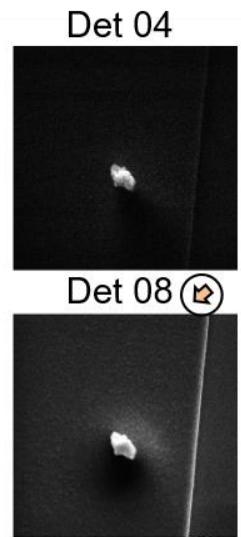

Det 12 ( )

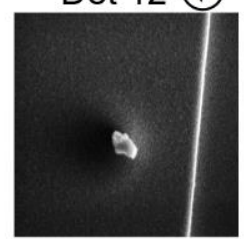

Det 16

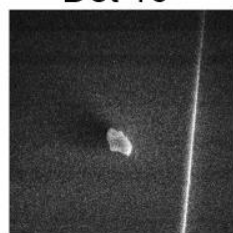

Figure 2. SEM images of a particle on Si pattern using the 16-channel detector.

\section{References}

[1] Otsuka et al., Journal Name 12., 279 (2014).

[2] Suzuki et al, Proc. Of SPIE 10145, 101451L-1 (2017). 